\title{
la Implantación del Grado en Relaciones Laborales y Recursos Humanos en la Facultad de Ciencias del Trabajo de la Universidad de Huelva. Cambios en los Sistemas de Organización Docente
}

\author{
Celia Sánchez López* \\ Yolanda Navarro Abal***
}

Resumen:

En este trabajo trataremos algunos de los cambios que ha generado la implantación del grado en RRLL y RRHH en la dinámica organizativa y cultural de los Centros (facultades y/escuelas universitarias), centrándonos especificamente en los cambios acaecidos en la Facultad de Ciencias del Trabajo de la Universidad de Huelva. En primer lugar haremos un breve recorrido histórico por la normativa que regula la construcción del EEES en general y el crédito ECTS en particular, en segundo lugar, analizaremos cómo se ha producido la adaptación de créditos LRU a crédito ECTS en las universidades andaluzas centrándonos además en aquéllas que han empezado con el grado en RRLL y RRHH y finalmente reflexionaremos sobre el impacto que el nuevo sistema de organización docente ha tenido sobre las dinámicas participativas del Centro tanto en lo relativo a los cambios en la metodologias de enseñanza-aprendizaje como en lo relativo a las dinámicas de organización docentes.

Palabras Clave:

Grado en Relaciones Laborales y Recursos Humanos, proceso de implantación del grado.

* Departamento de Economía. U. de Huelva • celia@ole.uhu.es

** Departamento de Psicología Clínica, Experimental y Social. U. de Huelva • yolanda.navarro@dpsi.uhu.es 


\section{ABstract:}

In this paper we discuss some of the changes that has led the implementation of the degree to RRLL and HR organizational dynamics and cultural centers (colleges and / colleges), focusing specifically on the Labor School University of Huelva. First we will take a brief historical overview of the regulations governing the construction of the EHEA in general and in particular ECTS credit, then, we analyze how the adaptation has to LRU ECTS credits in Andalusian universities also focus on those that have begun with the degree RRLL and HR and finally reflect on the impact that the new system has had on teaching organization on active dinamics of the Centre in terms of changes about the teaching-learning methodologies and in dynamical organization terms.

\section{KEYWORDS:}

Degree in Industrial Relations and Human Resources, degree implementation process.

\section{INTRODUCCIÓN}

Actualmente hay un total de 43 universidades españolas que ofertan titulaciones de grado de Relaciones Laborales (RRLL) y Recursos Humanos (RRHH), Relaciones Laborales, Relaciones Laborales y Desarrollo de RRHH, RRLL y Empleo, RRLL y Ocupación, Ciencias del Trabajo y RRHH y Ciencias del Trabajo ${ }^{1}$. No obstante, y pese que desde 1999 ya se establecen acuerdos para sentar las bases del nuevo Espacio de Europeo de Educación Superior en 2010, la realidad es que en 2008 sólo había una universidad con el título oficial (concretamente la Universidad Privada a Distancia de Madrid con el título de Ciencias del Trabajo y RRHH) y en 2009, había 18 universidades con titulaciones oficiales, 4 en trámite y 3 verificadas ${ }^{2}$.

La implantación de un Espacio Europeo de Educación Superior ha llevado asociado, y quizás por la inmediatez en la que se ha desarrollado el proceso, no sólo cambios en el mapa de titulaciones sino también importantes cambios en los sistemas organizativos docentes algunos de ellos sin la transparencia o sin la concreción adecuada. La adaptación del modelo tradicional de enseñanza — que salvo en excepciones en el ámbito universitario ha predominado el modelo transmisivo - al modelo de

1. Información extraída en diciembre de 2011 del Ministerio de Educación en la siguiente dirección web: http:// www.educacion.gob.es/educacion/universidades/educacion-superior-universitaria/que-estudiar-donde.html.

2. Información extraída de Galán García (2011:43) donde se analiza desde un punto de vista crítico y, a nuestro parecer muy realista, el proceso de implantación de los nuevos grados. 
enseñanza-aprendizaje que requiere el Espacio Europeo de Educación Superior (EEES) ha implicado grandes cambios tanto en la cultura organizativa y participativa como en los procesos de planificación y coordinación de la docencia universitaria. Algunos de estos cambios hacen referencia a: la forma en la que se organiza y planifica la docencia, la implicación de los estudiantes en su propio proceso de aprendizaje, los recursos humanos docentes necesarios para llevar a cabo el modelo, la flexibilidad y adaptabilidad de los horarios, la diversidad e incremento de los recursos didácticos así como en el manejo de los mismos y la dotación y disponibilidad de las infraestructuras que requiere el nuevo EEES, entre otros elementos. En este trabajo tratamos algunos de estos aspectos centrándonos específicamente en los cambios que ha generado la implantación del grado en RRLL y RRHH en la dinámica organizativa y cultural de nuestro Centro - la Facultad de Ciencias del Trabajo de la Universidad de Huelva-. En primer lugar hacemos un breve recorrido histórico por la normativa que regula la construcción del EEES, en general, y el crédito ECTS en particular, en segundo lugar, analizamos cómo se ha producido la adaptación de créditos LRU a crédito ECTS en las universidades andaluzas y los cambios que el proceso de implantación del grado en nuestro Centro $^{3}$ ha tenido sobre las dinamicas docentes, y finalmente, hacemos una reflexión sobre el impacto que la implementación del nuevo sistema de organización docente ha tenido sobre las dinámicas participativas del Centro tanto en lo relativo a los cambios en la metodologías de enseñanza-aprendizaje como en lo relativo a las dinámicas de organización docentes.

\section{CONTEXTO HISTÓRICO DEL PROCESO DE CONSTRUCCIÓN DEL EEES}

En la declaración de La Sorbona en 1998 se sientan las bases para la construcción de un sistema armonizado de Educación Superior en el ámbito europeo. En ella, los Ministros de Educación de los Estados de Francia, Alemania, Italia y Reino Unido acuerdan promover la convergencia entre los sistemas nacionales de educación superior con el objetivo, por un lado, de promover la movilidad geográfica de los ciudadanos y su empleabilidad, y por otro, favorecer y/o contribuir al desarrollo de las dimensiones cultural, intelectual, social, científica y tecnológica de la Unión en el marco del proceso europeo de convergencia ${ }^{4}$.

Con la construcción del EEES no se trata de someternos a un sistema o estructura con reglas establecidas sino de participar en la construcción de un marco común de enseñanza superior que permita la convalidación de los conocimientos adquiridos en

3. Un análisis del proceso de implantación del Grado en Relaciones Laborales y Recursos Humanos en la facultad de Ciencias del Trabajo de la Universidad de Huelva puede consultarse en Galán García (2011).

4. Aunque la iniciativa surgió en el marco del proceso de convergencia europea de mano de Reino Unido, Francia, Alemania e Italia que lideraron la declaración de La Sorbona, actualmente son más de 45 países implicados en el proceso (Plan de Acción 2009, p.2). 
alguna de las universidades europeas y facilite el reconocimiento de las titulaciones y de la formación adquirida. Esta idea se recoge en el siguiente fragmento extraído de la Declaración de La Sorbona de mayo de 1998:

"Un área europea abierta a la educación superior trae consigo una gran riqueza de proyectos positivos, siempre respectando nuestra diversidad, pero requiere, por otra parte, el esfuerzo continuo que permita acabar con las fronteras y desarrollar un marco de enseñanza y aprendizaje. Se espera que, de abora en adelante, éste favorezca una movilidad y cooperación más estrechas." (Declaración conjunta para la armonización del diseño del sistema de Educación Superior Europeo, La Sorbona mayo de 1998).

Posteriormente, en la Declaración de Bolonia en junio de 1999 se ratifican los principios establecidos en la declaración de la Sorbona y se establecen una serie de acuerdos con el objetivo principal de sentar de aquí a 2010 las bases del nuevo Espacio Europeo de Educación Superior. Los principales puntos contemplados en la Declaración hacen referencia:

- la adopción de un sistema de titulaciones sencillo, legible y comparable que favorezca la empleabilidad de los ciudadanos europeos y la competitividad internacional del Sistema Europeo de Enseñanza Superior - entre otros aspectos se propone implantar el suplemento europeo al títuló-,

- la implantación de un sistema de titulaciones de dos ciclos. El primer ciclo otorga cualificación en el mercado de trabajo europeo. El segundo ciclo llevará a la obtención de máster o doctorado,

- el establecimiento de un sistema de créditos que promueva la movilidad de los estudiantes y que además pueda ser adquirido, si está recogido en los planes de estudios de las universidades, en otros contextos - por ejemplo como formación permanente-,

- la eliminación de los obstáculos a la movilidad para estudiantes, profesores, investigadores y personal de administración y servicios,

- el fomento de la cooperación europea para asegurar un nivel de calidad para el desarrollo de criterios y metodologías comparables,

- la promoción de una necesaria dimensión europea de la enseñanza superior con especial atención del desarrollo curricular, colaboración institucional, planes de movilidad, etc. (Declaración de Bolonia, junio de 1999).

5. El Real Decreto 1044/2001 de 1 de agosto publicado en BOE el 11 de septiembre de 2003 recoge las características y el procedimiento de expedición del Suplemento Europeo al Título. 
En reuniones posteriores Praga (2001), Berlín (2003), Bergen (2005), Londres (2007) y Lovaina (2009) se reafirman los objetivos fijados para la creación de un Espacio Europeo de Educación Superior en 2010 insistiendo especialmente en aspectos como: la estructura de ciclos, el sistema de garantía de calidad, el marco de cualificaciones, la movilidad de estudiantes, personal académico y de administración y servicios, el reconocimiento de títulos y periodos de estudio, la formación a lo largo de la vida y los mecanismos de seguimiento de los progresos realizados en el marco del proceso de implementación del EEES, entre otros factores. En Lovaina (2009) se establecen, además, prioridades para la próxima década. Algunas de estas prioridades ya fueron recogidas en el plan de Bolonia pero fueron implantadas adecuadamente ${ }^{6}$.

En la declaración de Budapest-Viena en marzo de 2010, cuando da comienzo de forma oficial el Espacio Europeo de Educación Superior, se ratifica el compromiso acordado en Bolonia y se recoge el compromiso de atender las voces críticas que, en algunos países, se estaban levantando por parte del sector estudiantil y profesorado.

La próxima conferencia ministerial será organizada por Bucarest (Rumania) en abril del 2012.

Un elemento importante en el proceso de construcción del Espacio Europeo de Educación Superior lo constituye la introducción en el sistema universitario español del crédito ECTS ${ }^{7}$ (European Credits Transfer System). No sólo por la diferencias que éste presenta con respecto al significado de la noción de crédito seguido en el sistema universitario español, como veremos más adelante, sino también porque la adaptación del crédito LRU a crédito ECTS ha implicado importantes cambios en las dinámicas académicas de los centros y, en algunos centros, ha provocado una importante reducción de la carga docente del profesorado ante el mismo o mayor volumen de trabajo que el mantenido antes de la implantación del EEES ${ }^{8}$.

6. Algunas de las prioridades para la próxima década son: aumentar la formación continua adoptándose marcos nacionales de cualificaciones, proporcionar igualdad de oportunidades en el acceso a la enseñanza superior de cara a conseguir una mayor participación de los grupos infrarrepresentados, fomentar la movilidad, ligar la investigación y la innovación, mejorar la recogida de datos para el seguimiento y la evaluación de los avances perseguidos o, garantizar la financiación. Como se observa muchos de estos objetivos ya se contemplaban en el Plan de Bolonia. Para más información puede consultarse el comunicado de la conferencia de Ministros Europeos de Lovaina el 28-29 abril de 2009.

7. En el marco del Espacio Europeo de Educación Superior, el sistema de créditos europeos ECTS es el punto de partida para el desarrollo de los programas de movilidad de estudiantes, y por tanto garante de la interculturalidad, garantiza el reconocimiento académico de los estudios realizados en el extranjero. Véase los objetivos recogidos en la Declaración de Bolonia, página 3 de este trabajo.

8. No obstante, es necesario señalar que el Sistema Europeo de Transferencia de Créditos constituye un elemento clave de armonización a nivel europeo dado que, al poder generalizarse una misma unidad de medida a todos los estudiantes de la UE, se garantiza la transparencia necesaria de la formación, facilita el reconocimiento académico mediante la utilización de créditos y permite ajustar los programas docentes teniendo en cuenta al volumen de trabajo que éste genera para el alumno. 
En el sistema de educación superior en España, como en otros países europeos, la unidad de referencia sobre la que se estructuran los planes de estudio ha sido el crédito (en adelante crédito LRU). No obstante, existe un cambio importante con respecto al actual crédito europeo (en adelante crédito ECTS), mientras el crédito LRU venía definido en función de las horas de docencia, teóricas y/o prácticas, impartidas por los docentes, el crédito ECTS queda definido por el volumen de trabajo que el estudiante debe realizar para alcanzar los objetivos educativos, incluyéndose clases teóricas y/o actividades académicas dirigidas con o sin presencia del profesor y el esfuerzo dedicado al estudio y a la preparación de exámenes sin presencia directa del docente. De este modo, el crédito ECTS se define, según el Real Decreto como:

- "los créditos se computarán como boras de trabajo requeridas por el estudiante para adquirir conocimientos, capacidades y destrezas incluyendo boras lectivas (teóricas o prácticas), horas de estudio, horas dedicadas a la realización de seminarios y/o trabajos o proyectos y boras dedicadas a la preparación y realización de pruebas de evaluación.

- el cómputo total de créditos y la estimación de horas se hará partiendo de la base de una dedicación a tiempo completo del estudiante a la realización de los estudios. Entre 36 y 40 boras a la semana.

- la estimación del número de horas por créditos será entre 25 y 30 boras de trabajo." (RD. 1125/2003).

En síntesis, con el crédito ECTS la unidad de medida es el trabajo del estudiante mientras que con el crédito LRU la unidad de medida son las horas de clase u horas de docencia de los profesores. Este hecho, como veremos más adelante, supone un importante cambio en la planificación docente del profesorado y además carece de homogeneidad en su aplicación en las diferentes universidades andaluzas.

Entre los elementos que caracterizan los ECTS $^{9}$ destacamos especialmente dos por su relación con los objetivos que queremos cumplir en este trabajo: el primer aspecto a destacar es que el sistema de crédito europeo al construirse sobre la base del trabajo que supone para el alumno la preparación de la materia ha permitido mejorar los programas de estudio y la coordinación entre materias incluso de diferentes cursos, el segundo elemento que queremos tratar hace referencia a la propia definición del

9. Un análisis más detallado sobre el crédito europeo y la evolución de la implantación del mismo en el sistema educativo español puede consultarse en Pagani (2002). 
sistema $\mathrm{ECTS}^{10}$ por cuanto al representar el volumen de trabajo del estudiante y no las horas lectivas del docente, tal como se venía trabajando con anterioridad en España, existe una cierta indefinición de la proyección de estos créditos sobre la actividad docente del profesorado.

Estos dos aspectos son tratados en los siguientes apartados. En primer lugar analizamos, a partir de la experiencia adquirida durante estos años como docentes y coordinadores ECTS, los cambios organizativos docentes y las dinámicas participativas a las que ha dado lugar la implantación del nuevo grado en Relaciones Laborales y Recursos Humano en la Universidad de Huelva, y en segundo lugar, el proceso de conversión de créditos LRU a créditos ECTS en las Universidades Andaluzas.

\section{CAMBIOS EN LAS DINÁMICAS ORGANIZATIVAS DOCENTES}

La implantación del grado en Relaciones Laborales y Recursos Humano en la Facultad de Ciencias del Trabajo de la Universidad de Huelva, y por tanto nuestra adaptación al EEES, ha implicado cambios importantes tanto en los modelos de enseñanza-aprendizaje, como en los sistemas organizativos docentes universitarios y no sólo desde el punto de vista del docente como del discente. Si bien, es cierto que en el Centro contábamos con un bagaje importante adquirido con las experiencias piloto de implantación del crédito europeo en la diplomatura de Relaciones Laborales y en la Licenciatura en Ciencias del Trabajo ${ }^{11}$.

Por un lado, la adaptación de los créditos ECTS ha implicado unas guía didácticas (programas de las diferentes asignaturas) más viables, realistas, claras y mejor adaptadas a la realidad académica actual tanto en lo relativo al trabajo que genera cada materia para el estudiante como al grado de coordinación entre asignaturas de un mismo curso.

Muchos de los trabajos de coordinación no han venido impuestos ni desde los responsables de la coordinación académica ni desde el equipo de dirección del Centro

10. Según el Real Decreto 1125/2003 de 5 de septiembre en el Artículo 3 el crédito europeo se define como: "El crédito europeo es la unidad de medida del haber académico que representa la cantidad de trabajo del estudiante para cumplir los objetivos del programa de estudios y que se obtiene por la superación de cada una de las materias que integran los planes de estudios de las diversas enseñanzas conducentes a la obtención de títulos universitarios de carácter oficial y validez en todo el territorio nacional. En esta unidad de medida se integran las enseñanzas teóricas y prácticas, así como otras actividades académicas dirigidas, con inclusión de las horas de estudio y de trabajo que el estudiante debe realizar para alcanzar los objetivos formativos propios de cada una de las materias del correspondiente plan de estudios" (Real Decreto 1125/2003 de 5 septiembre. BOE núm. 224 18/09/2003 p. 34355).

11. Desde el curso 2004/2005 venimos trabajando en materia de organización docente en el Proyecto Piloto para la Implementación del Crédito Europeo en el primer curso de la Diplomatura. En la licenciatura en Ciencias del Trabajo la experiencia piloto comienza en el curso académico 2006/2007. 
sino que se han desarrollado en el seno de los propios equipos decentes. De hecho, la creación de equipos docentes ha sido uno de los principales cambios organizativos más importantes en lo relativo a la planificación de la docencia que se han llevado a cabo en el Centro. La coordinación aunque ha requerido mucho esfuerzo tanto por parte de la dirección del Centro como por parte de los docentes que integran los equipos docentes ha dado unos resultados espectaculares. Hoy en día, las fichas son perfectamente entendibles por los estudiantes y constituyen un importante elemento de consulta. Se ha conseguido unificar los criterios y metodologías de evaluación, y también algunas metodologías docentes, e incluso se han desarrollado actividades transversales en todas las asignaturas de un mismo curso coordinadas y evaluadas por los propios docentes. Actualmente la coordinación horizontal es mayor que la vertical. No obstante, seguimos trabajando en ello a medida que se produce la implantación del grado en cursos superiores.

Por otro lado, y desde el punto de vista del estudiante, y pese a que el perfil de alumnos/as está cambiando muy rápidamente, la implantación del créditos ECTS ha propiciado una mayor participación de los mismos en su propio proceso de aprendizaje y también en el ambiente universitario. Así, hay un alto porcentaje de alumnos que vuelven al centro para retomar su formación e incluso para seguir coparticipando en las actividades que se desarrollan en y desde el Centro.

También se está produciendo una mayor interacción profesor/a-alumno/a. De forma general destacamos algunos elementos de cambio:

- la función formativa ya no se dirige solamente a formar académicamente a jóvenes ciudadanos para su ingreso en el mundo laboral sino que ayuda a las personas que así lo requieran, a readaptar su desempeño profesional con nuevos aprendizajes que le permitan no estancarse profesional y/o laboralmente,

- las metodologías docentes están ahora más adaptadas a los perfiles de los alumnos, de hecho, es normal que en las aulas interaccionen discentes con características personales e inquietudes profesionales muy diferentes, más si cabe, en nuestro caso, donde se desarrollan titulaciones en tres modalidades: presencial, virtual y semipresencial,

- se han desarrollado importantes cambios en el sistema de enseñanza-aprendizaje en torno a las competencias que el discente debe adquirir tanto en lo relativo a las metodologías de trabajo como a los sistemas de evaluación. Se ha conseguido adaptar más las actividades docentes a los perfiles de los estudiantes. Si bien es cierto que es probable que llevemos a cabo una excesiva tutorización.

- cada asignatura desarrolla una metodología de trabajo diferente adaptada a las competencias que el alumno/a deba adquirir en su asignatura pero existen 
competencias que se trabajan de forma conjunta. De aquí, las actividades transversales que se desarrollan,

- se han llevado a cabo diferentes actividades transversales coordinadas entre diferentes materias de distintas áreas.

- se han unificado los programas de las asignaturas que ahora contienen información más precisa de los siguientes ítems ${ }^{12}$ : datos básicos de la asignatura en cuanto a número de créditos, cómputo en horas y tipo de asignatura; datos básicos de los profesores; descripción de la asignatura en cuanto a contenidos principales, requisitos y prerrequisitos, medidas previstas para responder a necesidades educativas especiales, sentido de la materia en la titulación, competencias, objetivos, metodología de trabajo, bloques temáticos y sistema de evaluación; organización del trabajo durante el curso, etc.

- actualmente en el seno de los equipos docentes también se han creado grupos de trabajo en diferentes proyectos de innovación y mejora de la actividad docente universitaria.

No obstante también es necesario destacar aquéllos aspectos que debemos mejorar como son: poner en valor la actividad docente, mejorar los cursos de formación del profesorado de tal modo que se adapten los requerimientos que exige el nuevo EEES y a las necesidades demandadas, y mejorar los sistemas de evaluación por competencias. Aspectos sobre los que estamos trabajando en la actualidad.

\section{ANÁLISIS DEL PROCESO DE ADAPTACIÓN DE CRÉDITOS LRU A CRÉDITO ECTS EN LAS UNIVERSIDADES ANDALUZAS}

En el proceso de implantación del EEES se ha pretendido mejorar la heterogeneidad y diversidad que caracterizaba los planes de estudios y programas universitarios no sólo de las universidades andaluzas sino también de las españolas ${ }^{13}$. No obstante, actualmente, y pese a que en aplicación al Acuerdo de la Comisión Académica del Consejo Andaluz de Universidades se impuso que al menos el 75\% de los contenidos de los grados debían ser comunes a todos los centros (incluidas las prácticas y el trabajo fin de grado), aún continúan existiendo importantes diferencias tanto en el sistema de organización de la docencia presencial como en el cómputo del trabajo docente en las diferentes universidades ante un mismo número de créditos ECTS.

12. A este respecto hay que destacar que durante el curso 2003/2004 la Facultad de Ciencias del Trabajo, participó en el Proyecto Piloto para la elaboración de la guía común de la Titulación de la Diplomatura de Relaciones Laborales en Andalucía.

13. Véase el informe sobre innovación de la docencia en las Universidades Andaluzas (2005). 
Así, aunque la normativa que define el sistema europeo de créditos y el sistema de calificaciones (RD. 1125/2003 de 5 de septiembre) establece una asignación específica de horas de trabajo del alumno por cada crédito ECTS - como anteriormente hacíamos referencia, entre un mínimo de 25 y un máximo de 30 horas de trabajo por crédito ECTS_- existen importantes diferencias tanto en las actividades que se desarrollan con o sin presencia del docente-como es el caso, como veremos más delante de las tutorías ${ }^{14}$ — , como en la distribución total de horas entre trabajo presencial y/o autónomo del alumno así como en el tamaño de los grupos a la hora de impartir la docencia que se promueve en el marco del Espacio Europeo de Educación Superior. En unos casos toda la docencia presencial se desarrolla en grandes grupos, en otros casos hay grupos grandes y pequeños como es el caso de Huelva y, en otros casos, hay grupos grandes, pequeños y medianos como se refleja en algunas fichas del grado (de RRLL y RRHH) en las distintas Universidades Andaluzas. No obstante, en éste último caso, y por la situación económica actual y la cantidad de recursos no sólo económicos sino también de infraestructuras y personal necesarios, entendemos que no es el momento adecuado para su estudio.

Si nos centramos en las características del Grado en Relaciones Laborales y Recursos Humanos en las Universidades Andaluzas ${ }^{15}$ y partiendo de una asignatura modelo de 6 créditos ECTS (150 horas de trabajo del alumno) el siguiente cuadro recoge la distribución de horas por tipo de actividad de una misma materia ${ }^{16}$ en las diferentes universidades andaluzas (véase tabla 1). Es necesario hacer constar que hay diferencias en la distribución de horas en las diferentes materias incluso en una misma Universidad.

Como se observa en la tabla, existen notables diferencias tanto en la distribución del número de horas destinadas a actividades académicas dirigidas por el docente - es decir, con presencia del docente- y el número de horas que el estudiante debe realizar sin presencia del docente para superar las asignaturas, como en el tipo de actividades académicas que son consideradas como horas presenciales u horas autónomas de trabajo del estudiante. Veamos estos dos aspectos más detenidamente.

14. Este hecho afecta especialmente al cómputo de las horas qiue forman parte de plan de ordenación docente. de hecho, como hace referencia el profesor Galán (2011:33) en la mayor parte de las universidades las asignaturas se ha seguido considerando, a falta de un Reglamento de Régimen de dedicación del profesorado universitario, como de créditos LRU a los que se le ha añadido la parte correspondiente a las horas dedicadas al trabajo en grupos reducidos e incluso dedicado a las tutorías individualizadas.

15. Para realizar este trabajo hemos consultado las fichas de las diferentes asignaturas de primero del Grado en Relaciones Laborales y Recursos Humanos en las distintas Universidades Andaluzas del curso 2010/2011a partir de la información contenida en sus páginas webs.

16. En este caso, y de forma aleatoria, hemos seleccionado la asignatura de Administración de Empresas. 
Tabla 1. Distribución de horas de trabajo presencial y no presencial del estudiante EQUIVALENTE A 6 ECTS.

\begin{tabular}{lccccccccc}
\hline & $\begin{array}{c}\text { U. Pablo } \\
\text { de } \\
\text { Olavide }\end{array}$ & $\begin{array}{c}\text { U. } \\
\text { Sevilla }\end{array}$ & $\begin{array}{c}\text { U. } \\
\text { Huelva }\end{array}$ & $\begin{array}{c}\text { U. } \\
\text { Cádiz }\end{array}$ & $\begin{array}{c}\text { U. } \\
\text { Granada }\end{array}$ & $\begin{array}{c}\text { U. } \\
\text { Jaén }\end{array}$ & $\begin{array}{c}\text { U. } \\
\text { Málaga }\end{array}$ & $\begin{array}{c}\text { U. } \\
\text { Almería }\end{array}$ & $\begin{array}{c}\text { U. } \\
\text { Córdoba }\end{array}$ \\
Presenciales & 45 & 60 & 45 & 60 & 45 & 60 & 45 & 45 & 60 \\
No presenciales & 90 & 90 & 105 & 90 & $87,5^{18}$ & 90 & 90 & 105 & 90 \\
Evaluación & 15 & & & & & & & & \\
Tutorías & 150 & 150 & 150 & 150 & 152,5 & 150 & 150 & 150 & 150 \\
\hline Total & & & & & & & & & \\
\hline
\end{tabular}

A grandes rasgos se observan tres tendencias diferenciadas en cuanto a la distribución del número total de horas de trabajo del estudiante entre horas de trabajo presencial y horas de trabajo autónomo para una misma asignatura de 6 créditos ECTS:

- en primer lugar, aquellas universidades que optan por una distribución del número total de horas de trabajo del estudiante entre presencial y no presencial del 40\%/60\%, es decir, 60 horas de trabajo presencial del estudiante y 90 horas de trabajo autónomo. En este caso, 1 asignatura de 6 créditos ECTS equivale al mismo tiempo que una asignatura de 6 créditos LRU. En esta situación se encuentran, según el análisis de la información contenidas en las fichas analizadas, las Universidades de Sevilla, Cádiz, Jaén y Córdoba (véase tabla 1).

- en segundo lugar, aquellas otras universidades que han optado por una distribución del número total de horas de trabajo del estudiante entre presencial y no presencial del 30\%-70\%, es decir, 45 horas de trabajo presencial del estudiante con el docente frente a 105 horas de trabajo autónomo- como es el caso de

17. Sólo en algunas fichas de las consultadas estaba disponible esta información. El porcentaje de horas dedicadas a trabajo autónoma es aproximadamente del 60\%, entre 5-10\% a evaluación y entre 35-30\% a docencia presencial.

18. El hecho de que se obtenga en este caso un total de créditos de 87,5 es debido a que, según el informe técnico "El crédito europeo y el sistema educativo español" el esfuerzo del alumno equivalente a una hora de sesión teórica es de 1,5. Por tanto 45 horas de sesiones teóricas equivalen a 87,5 horas de trabajo autónomo del estudiante. Véase Pagani (2002: 26 y ss.).

19. En este caso aunque la norma general es que la metodología a seguir en la docencia de la materia constará de un 30\% de docencia presencial en el aula, es decir, 45 horas, un 60\%, es decir, 90 horas para estudio y la búsqueda, consulta, tratamiento de la información, experimentación y observación necesarias para la realización de trabajos e informes de campo o de laboratorio, y un 10\%, es decir, 15 horas para tutorías colectivas e individuales (presenciales o virtuales) y evaluación, no existe homogeneidad entre los ítems de las diferentes asignaturas. En unos casos las 15 horas son tutorías individuales o colectivas, en otros casos son horas de resolución de problemas, en otros casos se reparte entre varios ítems, etc. 
las Universidades de Almería y Huelva. En este caso, 1 asignatura de 6 créditos ECTS le computa al profesor como 4,5 créditos LRU, y finalmente,

- en tercer lugar, están aquellas universidades que han optado por una distribución del 30\% presencial, 60\% de trabajo autónomo del estudiante y 10\% de tutorías/exposiciones/evaluación, como es el caso de las Universidades de Pablo de Olavide, Málaga y Granada. En este caso el cómputo total de horas para el profesor depende de si ese 10\% restante es considerado como horas con presencia del profesor por el centro o no.

Por otro lado, el segundo aspecto a analizar era el tipo de actividades académicas que son consideradas como horas presenciales u horas autónomas de trabajo del estudiante. Si analizamos la distribución y organización de la docencia por tipo de actividad en las distintas universidades andaluzas — véase anexo I- se observa una gran diversidad fruto del esfuerzo de la comunidad universitaria por adaptarse a los nuevos requerimientos que exige el EEES. Mientras en algunas guías docentes ${ }^{20}$ sólo se especifica la impartición de sesiones teóricas y prácticas de forma global y en ambos casos el número total de horas se computan como actividades de carácter presencial, en otras, se observa una mayor diversidad de técnicas metodológicas y su encuadre como actividades presenciales o no presenciales es más heterogéneo. Así, si tomamos el número de horas de tutorías individuales y/o colectivas en unos casos éstas se computan como horas presenciales del alumno pero fuera del 30\%/40\% de presencialidad del estudiante, como es el caso de la Universidad de Cádiz o la Universidad de Málaga y, en los otros, las horas de tutorías quedan fuera de las horas de trabajo presencial del estudiante como es el ejemplo de la Universidad de Sevilla, y en otras ocasiones, las horas de tutorías resultan el 10\% restante después de la distribución de horas del estudiante en presencialidad y no presencialidad de 30\%-60\% como es el caso de la Universidad de Granada (véase anexo 1).

En el caso del grado en Relaciones Laborales y Recursos Humanos de la Universidad de Huelva, se ha optado por 25 horas de trabajo del alumno por crédito ECTS, una presencialidad del 30\% sobre el cómputo total de horas de trabajo de cada materia ( 45 horas de las 150 horas que corresponden a 6 créditos ECTS) y como metodología de trabajo presencial se imparte el 80\% de la docencia presencial en gran grupo ( 45 horas) (inicialmente 65 alumnos por curso) y el 20\% en pequeños grupos de un máximo de 25-30 personas. El siguiente esquema reproduce en líneas generales la metodología de trabajo en el aula seguida en nuestro centro y consensuada por todos los profesores/as que imparten docencia en primero de grado.

20. Se han revisado tanto los planes de estudios como las guías docentes de las asignaturas de las titulaciones de Grado en Relaciones Laborales y Recursos Humanos disponibles en internet de las diferentes Universidades Andaluzas referentes al curso académico 2010/2011. 
Tabla 2. Distribución del número de horas de trabajo Del alumno.

\begin{tabular}{lc} 
& NúMERO DE \\
CON SUPERVISIÓN DEL PROFESOR & $\mathbf{4 5}$ \\
\hline Clases teórico-prácticas en gran grupo & 32.5 \\
\hline Clases prácticas en grupo reducido & 10 \\
\hline Realización de Exámenes & 2.5 \\
\hline Otras (especificar) Actividades transversales & $\mathbf{1 0 5}$ \\
\hline TRABAJO PERSONAL DEL ALUMNO. ACTIVIDADES DIRIGIDAS Y ESTUDIO & \\
\hline Elaboración de trabajos, ejercicios u otras actividades dirigidas & \\
\hline Programación/Experimentación u otros trabajos con ordenador & $\mathbf{1 5 0}$ \\
\hline Lecturas recomendadas, actividades en biblioteca o similar & \\
\hline Preparación de presentaciones orales, debates o similar & \\
\hline Asistencia a charlas, exposiciones u otras actividades recomendadas & \\
\hline № TOTAL DE HoRAS & \\
\hline
\end{tabular}

No obstante, aunque consensuamos unos parámetros comunes básicos, son muchas y muy diversas las actividades que se llevan a cabo en el aula. Un hecho novedoso durante este año académico ha sido la realización de actividades conjuntas transversales coordinadas por las diferentes asignaturas desarrolladas en el marco de los contenidos de las mismas y el establecimiento de un sistema común y consensuado de evaluación.

Desde el punto de vista del trabajo que genera para el docente la nueva implantación del crédito ECTS frente al crédito LRU ha de decirse que ha generado más rechazo que aceptación porque si bien la coordinación "gratuita" conseguida en este proceso ha hecho mejorar considerablemente la calidad de la docencia, el trabajo para el docente es mayor. Una asignatura de 6 LRU computaba para el profesor 6 créditos - 60 horas de sesiones presenciales - y ahora una asignatura de 6 créditos ECTS que genera más trabajo computa para el profesor 5, 4 créditos - 54 horas de sesiones presenciales - resultante de 45 horas de clases teórico-prácticas en grupo grande y 9 horas de trabajo en pequeño grupo.

\section{REFLEXIONES}

Es obvio que la implantación de los nuevos modelos y estructuras docentes han traído como consecuencia aspectos positivos y negativos. Aunque, dado la coyuntura económica en que nos encontramos, los aspectos negativos prevalecen existiendo una sensación generalizada de percibir que esta nueva estructura docente se ha im- 
plementado gracias al esfuerzo de los profesores y en algunos casos, hay que decir, es totalmente cierto.

Así, por ejemplo, las enseñanzas según programas docentes basados en la transferencia del conocimiento y el desarrollo de otras competencias profesionales -objetivo prioritario en el Plan Bolonia-, llevaban implícitas un número de alumnos por grupo más reducido, que permitiera el trabajo en equipo y el fomento de otras habilidades y destrezas. La realidad no ha sido así y, en muchas titulaciones, los grupos grandes se van a conformar por grupos constituidos por 75 alumnos y grupos pequeños de 35 o 40. Esta cifra, evidentemente, limita la metodología a aplicar, obligando a retomar prácticas docentes usadas anteriormente a este nuevo modelo de enseñanza-aprendizaje o a un sobreesfuerzo del docente, sin poder asegurar que la calidad pueda ser la misma. Por tanto, la exigencia del espacio europeo de generar contextos en donde resulte fácil el trabajo de actividades teórico-prácticas se vuelve una utopía sin olvidar que todas las actividades realizadas por el alumnado deben pasar por un proceso de evaluación que debe realizar el docente, que ve como se multiplican las actividades y su cómputo docente y su reconocimiento curricular sigue siendo el mismo.

Otro ejemplo, en el cual se evidencia la falta de concreción y homogeneidad en las diferentes universidades en relación a los distintos aspectos el EEES, se observa en la relevancia otorgada a la figura del coordinador/a de los equipos docentes. De esta forma, se encuentran ejemplos contrapuestos, en algunas universidades no existe esta figura y en otras tiene funciones propias de un vicedecanato. En algunos casos, la coordinación es remunerada o exonerada mientras que en otras ocasiones no existe ninguna consideración del cargo. Esta circunstancia lleva implícita el mayor o menor reconocimiento por parte de los propios compañeros. Como consecuencia se le otorga un mayor o menor estatus y autoridad y, a su vez, este hecho influye en la capacidad del mismo de poder gestionar y liderar los equipos docentes.

Así mismo, se pueden analizar los procedimientos llevados a cabo en cuanto a equipos docentes. No aparecen claros criterios consensuados y en la mayoría de las ocasiones no hay sistemas de sanción para aquellas personas que sistemáticamente obvian la existencia de estos equipos y fomentan el abandono en la participación de otras personas que ven como "la participación o no" no implica un mayor reconocimiento. Así mismo, tampoco parece haber un consenso en la adecuación o no del número de reuniones, objetivos de las mismas, etc. También es conveniente tener en cuenta que, el profesorado, a veces, no se implica suficientemente en los equipos docentes ya que la planificación de la asignatura que va a impartir y la coordinación con otras asignaturas y profesores conlleva un mayor trabajo y sobrecarga y, puede ocurrir, que al año siguiente pueda no impartir la misma asignatura si algún compa- 
ñero con preferencia en el orden de prelación decide seleccionarla. Así que después del trabajo realizado, sabe que podría tener que volver nuevamente a prepararse y planificar otra asignatura. Sería conveniente establecer un sistema de control que regulara la permanencia de los docentes en asignaturas que presentan un buen funcionamiento y no dejarlo "al capricho del más antiguos". Así también favorecería la creación de asignaturas más consolidadas y planificadas.

Para finalizar sería conveniente hacer una reflexión sobre las funciones del docente universitario en estos últimos años. La evaluación del desempeño del mismo se basa en tres pilares fundamentales, docencia, investigación y gestión. Cada uno de estos ejes ha requerido constantemente de procesos de arduo trabajo que se agravan por la sobrecarga de comisiones y burocratización que dificultan la productividad del docente en alguna de las áreas a evaluar. En este período de implementación se han producido grandes cambios en procesos determinantes para el docente, tales como las agencias de acreditación, los programas docentia, las nuevas guías docentes, los nuevos procesos de enseñanza-aprendizaje virtuales. Además, se han visto agravados por decisiones políticas como los recortes salariales. Todos ellos han ido constituyendo estresores cotidianos de la vida del docente dando como resultado que las instituciones educativas de grado superior se encuentren conformadas por un alto número de profesionales que manifiestan altos niveles de estrés (síndrome de burnout). Este hecho trae consecuentemente una organización no saludable conformada por miembros con patologías psicosomáticas, un mal clima laboral, y evidentemente, una peor productividad y una menor calidad. Quizás en los años próximos sería necesario detenerse y tomar conciencia de estas circunstancias y comenzar a generar un contexto laboral que propicie el bienestar de los trabajadores y, en consecuencia, una educación de más calidad.

\section{BIBLIOGRAFIA}

Informe de la Comisión para la Innovación de la Docencia en las Universidades Andaluzas (CIDUA) (2005). Dirección General de Universidades, Consejería de Innovación, Ciencia y Empresa. Disponible en: http://www.uma.es/eees/

Declaraciones conjuntas y comunicados de ministros europeos de enseñanza:

- Declaración conjunta para la armonización del diseño del Sistema de Educación Superior Europeo, la Sorbona, París, 25 de mayo de 1998.

- Declaración conjunta sobre el Espacio Europeo de Enseñanza Superior, Bolonia, 19 de junio de 1999.

- Declaración conjunta del encuentro de Ministros Europeos en funciones de la educación Superior hacia el área de la Educación Superior Europea, Praga, 19 de mayo de 2001. 
- Comunicado de la Conferencia de Ministros responsables de la Educación Superior sobre "Educación Superior Europea", Berlín, 19 de septiembre de 2003.

- Comunicado de la Conferencia de Ministros Europeos responsables de la Educación Superior sobre "El Espacio Europeo de Educación Superior-Alcanzando las metas", Bergen, 19-20 de mayo de 2005.

- Comunicado de la Conferencia de Ministros Europeos responsables de la Educación Superior sobre "Hacia el Espacio Europeo de Educación Superior: respondiendo a los desafíos de un mundo globalizado", Londres, 18 de mayo de 2007.

- Comunicado de la Conferencia de Ministros Europeos responsables de la Educación Superior sobre "El proceso de Bolonia 2020- la nueva década del Espacio Europeo de Educación Superior”, Leuven and Lovain-la-Neuve, 28-29 de abril de 2009.

Galán García, A. (2011): "El proceso de convergencia desde el Grado de Relaciones Laborales y Recursos Humanos: una ojeada al panorama nacional", Martín, M. y Roldán, A., EEES y cambios en las Metodologías Docentes. Reflexiones y Experiencias en su aplicación a las Ciencias del Trabajo, Valencia, Tirant lo Blanch, pp.15-45.

Ministerio de Ciencia e Innovación (2009): Plan de Acción 2009 para el Apoyo y la Modernización de la Universidad Pública Española en el Marco de la Estrategia Universidad 2015, Madrid, Ministerio de Educación.

Pagani, R. (2002): El crédito Europeo y el Sistema Educativo Español, Informe Técnico, ECTS Counsellor \& Diploma Supplement Promoter, Madrid 20 de septiembre de 2002.

Real Decreto 1044/2003, de 1 de agosto, por el que se establece el procedimiento para la expedición por las universidades del Suplemento Europeo al Título. Publicado el 11 de septiembre de 2003. 
ANEXO I.

METODOLOGÍA

\begin{tabular}{|c|c|c|c|c|c|}
\hline \multirow{5}{*}{$\begin{array}{l}\text { Universidad de } \\
\text { Sevilla }\end{array}$} & \multirow{5}{*}{$\begin{array}{l}\text { Administración } \\
\text { de empresas }\end{array}$} & $\begin{array}{c}\text { Horas } \\
\text { presenciales }\end{array}$ & 60 & Clases teóricas & 25 \\
\hline & & & & Clases prácticas & 35 \\
\hline & & & 90 & Exposiciones y seminarios & 30 \\
\hline & & $\begin{array}{l}\text { No } \\
\text { presenciales }\end{array}$ & & Trabajo de investigación & 20 \\
\hline & & & & Exámenes & 40 \\
\hline
\end{tabular}

\begin{tabular}{|c|c|c|c|c|c|}
\hline \multirow{5}{*}{$\begin{array}{l}\text { Universidad de } \\
\quad \text { Cádiz }\end{array}$} & \multirow{5}{*}{$\begin{array}{l}\text { Administración } \\
\text { de empresas }\end{array}$} & \multirow{4}{*}{ Presenciales } & 60 & Clases teóricas & 32 \\
\hline & & & & Clases prácticas/seminarios & 16 \\
\hline & & & & Tutorías individuales/colectivas & 6 \\
\hline & & & & Actividades de evaluación & 6 \\
\hline & & $\begin{array}{c}\text { No } \\
\text { presenciales }\end{array}$ & 90 & - & \\
\hline \multirow{7}{*}{$\begin{array}{l}\text { Universidad de } \\
\text { Granada }\end{array}$} & \multirow{7}{*}{$\begin{array}{l}\text { Administración } \\
\text { de empresas }\end{array}$} & \multirow{4}{*}{ Presenciales } & 65 & Clases teóricas & 45 \\
\hline & & & & Clases problemas & 15 \\
\hline & & & & Seminarios y/o exposiciones & 4 \\
\hline & & & & Exámenes & 1 \\
\hline & & \multirow{3}{*}{$\begin{array}{c}\text { No } \\
\text { presenciales }\end{array}$} & 87,5 & Clases teóricas & 67,5 \\
\hline & & & & Clases problemas & 15 \\
\hline & & & & Preparación trabajos & 5 \\
\hline \multirow{4}{*}{$\begin{array}{l}\text { Universidad de } \\
\text { Jaén }\end{array}$} & \multirow{4}{*}{$\begin{array}{l}\text { Administración } \\
\text { de empresas }\end{array}$} & \multirow{2}{*}{ Presenciales } & 60 & \multirow{2}{*}{$\begin{array}{l}\text { Clases expositivas en gran grupo } \\
\text { Clases en grupos de prácticas }\end{array}$} & 45 \\
\hline & & & & & 15 \\
\hline & & \multirow{2}{*}{$\begin{array}{l}\text { No } \\
\text { presenciales }\end{array}$} & 90 & \multirow{2}{*}{$\begin{array}{l}\text { Clases expositivas en gran grupo } \\
\text { Clases en grupos de prácticas }\end{array}$} & 120 \\
\hline & & & & & 30 \\
\hline \multirow{3}{*}{$\begin{array}{c}\text { Universidad } \\
\text { Pablo de Olavide }\end{array}$} & \multirow{3}{*}{$\begin{array}{l}\text { VER QUÉ } \\
\text { ASIGNATURA }\end{array}$} & Presenciales & 45 & & \\
\hline & & $\begin{array}{c}\text { No } \\
\text { presenciales }\end{array}$ & 90 & & \\
\hline & & Evaluación & 15 & & \\
\hline $\begin{array}{l}\text { Universidad de } \\
\text { Málaga }\end{array}$ & $\begin{array}{l}\text { Administración } \\
\text { de empresas }\end{array}$ & $\begin{array}{l}\text { Presenciales } \\
\text { No } \\
\text { presenciales } \\
\text { Otras }\end{array}$ & $\begin{array}{l}45 \\
90 \\
15\end{array}$ & $\begin{array}{l}\text { docencia presencial } \\
\text { horas de estudio } \\
\text { Tutorías individuales/colectivas }\end{array}$ & $\begin{array}{l}45 \\
90 \\
15\end{array}$ \\
\hline \multirow{2}{*}{$\begin{array}{l}\text { Universidad de } \\
\text { Almería }\end{array}$} & \multirow{2}{*}{$\begin{array}{l}\text { Administración } \\
\text { de empresas }\end{array}$} & Presenciales & 45 & $\begin{array}{l}\text { Horas presenciales del } \\
\text { estudiante }\end{array}$ & 45 \\
\hline & & $\begin{array}{c}\text { No } \\
\text { presenciales }\end{array}$ & 105 & $\begin{array}{l}\text { Horas no presenciales del } \\
\text { estudiante }\end{array}$ & 105 \\
\hline
\end{tabular}




\section{METODOLOGÍA (continuación)}

\begin{tabular}{|c|c|c|c|c|c|}
\hline \multirow{5}{*}{$\begin{array}{c}\text { Universidad de } \\
\text { Córdoba }\end{array}$} & \multirow{5}{*}{$\begin{array}{c}\text { Administración } \\
\text { de empresas }\end{array}$} & \multirow{4}{*}{ Presenciales } & 60 & Clases magistrales & 40 \\
\hline & & & & Estudio de casos & 15 \\
\hline & & & & Actividades de evaluación & 3 \\
\hline & & & & Tutorías individuales/colectivas & 2 \\
\hline & & $\begin{array}{c}\text { No } \\
\text { presenciales }\end{array}$ & 90 & & 90 \\
\hline \multirow{4}{*}{$\begin{array}{c}\text { Universidad de } \\
\text { Huelva }\end{array}$} & \multirow{4}{*}{$\begin{array}{l}\text { Administración } \\
\text { de empresas }\end{array}$} & \multirow{3}{*}{ Presenciales } & 45 & Clases teórico/prácticas & 36 \\
\hline & & & & Clases prácticas/seminarios & 8 \\
\hline & & & & Actividades transversales & 1 \\
\hline & & $\begin{array}{c}\text { No } \\
\text { presenciales }\end{array}$ & 105 & Trabajo autónomo & 105 \\
\hline
\end{tabular}

